\title{
Diastolic tolerance to systolic pressures closely reflects systolic performance in patients with coronary heart disease
}

\author{
Adelino F. Leite-Moreira • André P. Lourenço • \\ Roberto Roncon-Albuquerque Jr. • Tiago Henriques-Coelho • \\ Mário J. Amorim · Jorge Almeida • Paulo Pinho • \\ Thierry C. Gillebert
}

Received: 24 November 2011/Revised: 5 January 2012/ Accepted: 26 January 2012/Published online: 5 February 2012

(c) Springer-Verlag 2012

\begin{abstract}
In animal experiments, elevating systolic pressures induces diastolic dysfunction and may contribute to congestion, a finding not yet translated to humans. Coronary surgery patients ( $63 \pm 8$ years) were studied with left ventricular $(\mathrm{LV})$ pressure $(n=17)$ or pressure-volume $(n=3)$ catheters, immediately before cardiopulmonary bypass. Single-beat graded pressure elevations were induced by clamping the ascending aorta. Protocol was repeated after volume loading $(n=7)$. Consecutive patients with a wide range of systolic function were included. Peak isovolumetric $\mathrm{LV}$ pressure $\left(\mathrm{LVP}_{\mathrm{iso}}\right)$ ranged from 113 to $261 \mathrm{mmHg}$. With preserved systolic function, LVP elevations neither delayed relaxation nor increased filling pressures. With decreasing systolic function, diastolic tolerance to afterload progressively disappeared: relaxation slowed and filling pressures increased (diastolic dysfunction). In severely depressed systolic function, filling pressures increased even with minor LVP elevations, suggesting baseline load-dependent elevation of diastolic pressures. The magnitude of filling pressure elevation induced in isovolumetric heartbeats was closely and inversely related to systolic performance, evaluated by
\end{abstract}

Electronic supplementary material The online version of this article (doi:10.1007/s00395-012-0251-y) contains supplementary material, which is available to authorized users.

A. F. Leite-Moreira $(\bowtie) \cdot$ A. P. Lourenço ·

R. Roncon-Albuquerque Jr. · T. Henriques-Coelho ·

M. J. Amorim - J. Almeida · P. Pinho

Cardiovascular R\&D Unit, Department of Physiology and Cardiothoracic Surgery, Faculty of Medicine, Hospital São João,

Universidade do Porto, Alameda Professor Hernâni Monteiro,

4200-319 Porto, Portugal

e-mail: amoreira@med.up.pt

T. C. Gillebert

Department of Cardiology, University of Ghent, Ghent, Belgium
$\operatorname{LVP}_{\text {iso }}(r=-0.96)$, and directly related to changes in the time constant of relaxation $\tau(r=0.95)$. The maximum tolerated systolic LVP (without diastolic dysfunction) was similarly correlated with $\mathrm{LVP}_{\text {iso }}(r=0.99)$. Volume loading itself accelerated relaxation, but augmented afterloadinduced upward shift of filling pressures $(7.9 \pm 3.7$ vs. $3.0 \pm 1.5 ; P<0.01)$. The normal human response to even markedly increased systolic pressures is no slowing of relaxation and preservation of normal filling pressures. When cardiac function deteriorates, the LV becomes less tolerant, responding with slowed relaxation and increased filling pressures. This increase is exacerbated by volume loading.

Keywords Diastole · Diastolic dysfunction · Afterload · Systolic function

\section{Introduction}

Ventricular remodelling in coronary heart disease includes myocyte loss, changes in myocyte biology and extracellular matrix, and alterations in chamber geometry. These aspects contribute to diastolic dysfunction [13,29], which is characterized by impaired ventricular filling and an upward shift of the diastolic pressure-volume relation [14, 20]. The main cause of diastolic dysfunction is increased late-diastolic stiffness $[5,12,15]$. Accumulating evidence shows that, in addition to long-term structural changes that underlie myocardial stiffness [11, 38, 40], there might also be short-term functional determinants such as ischemia [33], titin phosphorylation status [2] and neuroendocrine mediation [23].

Impaired myocardial relaxation may result in sustained pressure at end-diastole and may thus contribute to increased left ventricular (LV) stiffness, mainly in failing 
hearts [12]. One of the possible causes for impaired myocardial relaxation and diastolic dysfunction in animal models is excessive afterload [25]. This load-dependence might be relevant as well for the interpretation of diastolic dysfunction resulting from arterial hypertension, from increased arterial stiffening and from early wave reflection [3]. In order to clarify the relevance of these concepts to human disease, the present study analysed the effects on diastolic filling pressures of graded elevations of systolic LV pressures (LVP) induced by aortic clamping. The study was performed during coronary artery bypass grafting (CABG).

\section{Materials and methods}

\section{Study population}

Twenty consecutive adult patients with 3-vessel disease undergoing elective on-pump CABG were enrolled. Exclusion criteria included: unstable angina, pericardial disease, LV hypertrophy defined as mean wall thickness $>1.1 \mathrm{~cm}$, evidence of calcified ascending aorta in preoperative exams or in intra-operative assessment both by palpation and epivascular ultrasonography, previous stroke or transient ischemic event, as assessed by clinical interview or preoperative exams, significant carotid artery disease based on preoperative evaluation, and previous cardiac surgery. All patients underwent routine preoperative evaluation including coronary angiogram and echocardiographic evaluation. Left ventricular ejection fraction (EF) was calculated by 2D-echocardiography using the Simpson's rule. The study was approved by the ethics committee of the University Hospital São João in Porto and conforms with the principles of the Declaration of Helsinki. All patients gave their written informed consent.

\section{Procedure}

Preoperative, anaesthetic and surgical procedures were standard. Briefly, regular medication was continued until the morning of surgery, $0.1 \mathrm{mg} / \mathrm{kg}$ oral diazepam was used as an anxiolytic in the morning and on the night before the intervention. On arrival to the operation room, the EKG and pulse oximetry were monitored. Patients were then premedicated with $0.1 \mathrm{mg} / \mathrm{kg}$ intravenous diazepam, and invasive blood pressures were monitored after radial artery catheterization under local anaesthesia. General anaesthesia was induced with $10-25 \mu \mathrm{g} / \mathrm{kg}$ fentanyl, $0.1 \mathrm{mg} / \mathrm{kg}$ etomidate and $0.1 \mathrm{mg} / \mathrm{kg}$ vecuronium, and maintained with $0.2-0.4 \%$ isoflurane on a $50 \% \mathrm{O}_{2}: \mathrm{N}_{2} \mathrm{O}$ gas mixture, and additional fentanyl and vecuronium boluses. A central venous line was then placed and central venous pressure was continuously monitored, as well as capnography, central and peripheral temperature. An additional bolus of $20-25 \mu \mathrm{g} / \mathrm{kg}$ fentanyl was given before sternotomy. Before anaesthesia mean blood pressure was $86.4 \pm 12.0 \mathrm{mmHg}$ and decreased to $77.7 \pm 13.2 \mathrm{mmHg}$ at the start of the measurements. During surgical preparation for cardiopulmonary bypass, just before the insertion of the venous cannula, a 3F catheter with a high-fidelity transducer (SPC330A, Millar Instruments, Houston, TX, USA) was inserted through a needle puncture in the LV apical dimple and held in place by a Teflon felt pledgeted purse-string suture with $4 / 0$ polypropylene in order to record LV pressures. Before insertion, the catheter was calibrated and stabilized for $30 \mathrm{~min}$ in $37^{\circ} \mathrm{C}$ saline. The catheter was connected to a pressure amplifier and a differentiator to evaluate the first derivative of the pressure recording. To strengthen our findings, in three patients we simultaneously recorded LV pressure and volume using an equipment transiently available at our institution. For this purpose a 5-Fr combined pressure-volume catheter with $1-\mathrm{cm}$ inter-electrode spacing (SPC-551, Millar Instruments, Houston, TX, USA) was inserted instead.

\section{Data acquisition and analysis}

EKG (DII), LV pressure and its first derivative were digitized with a sample rate of $500 \mathrm{~Hz}$. The mean heart rate (HR) value was $72 \pm 5 \mathrm{bpm}$ and remained stable. Haemodynamic recordings were done with ventilation suspended at end-expiration. The $\mathrm{R}$ wave of the EKG tracing was used to define end-diastole. The following parameters were obtained: LV end-diastolic pressure (LVEDP), peak systolic LVP, maximum velocity of $\mathrm{LV}$ pressure rise $(\mathrm{d} P /$ $\left.\mathrm{d} t_{\max }\right)$ and fall $\left(\mathrm{d} P / \mathrm{d} t_{\min }\right)$, and the logistic time constant of isovolumetric relaxation $\tau[30,37]$.

Beat-to-beat systolic LVP elevations were induced by constricting the ascending aorta with an aortic clamp, above the sinotubular junction. Variable degrees of constriction were performed and isovolumetric heartbeats were obtained with complete aortic occlusions. Constrictions were started during diastole and sustained for $2-5$ cycles. An interval of 2-3 min of rest and stabilization was observed between manoeuvers. No electrocardiographic signs of ischemia or haemodynamic instability were observed before, during or after the interventions. The first heartbeat after the clamp was analysed. From physiology and previous experiments in animal models, we know that aortic clamps increase systolic pressure and systolic volume while decreasing systolic wall thickness. The three parameters of Laplace's law concur to increase systolic wall stress and hence afterload. In the given experimental conditions, systolic pressure can therefore be considered a surrogate of afterload. The clamp technique selectively 
increases afterload with no preload changes, no changes in long-term load history, and no neurohumoral adaptations [26].

Effects of systolic LVP elevations on filling pressures were assessed by subtracting LVEDP at the end of the test beat from LVEDP of the previous control beat (diastolic dysfunction). We previously showed in animal experiments that single beat afterload elevations do not alter LV enddiastolic volume and therefore increases in LVEDP denote a true upward shift of the end-diastolic pressure-volume relation [25]. This was confirmed in the present study with pressure-volume measurements.

In each patient the maximum systolic LVP, which did not slow relaxation and did not increase subsequent filling pressures was determined, referred to as maximum tolerated pressure. In three patients, in whom no additional systolic LVP could be developed without slowing of relaxation, systolic pressure was carefully decreased by transient caval occlusion. As LVP progressively decreased, the time constant $\tau$ initially decreased, then increased. This was monitored online on a $\mathrm{d} P / \mathrm{d} t$ versus LVP phase-plane plot, as previously described [26], and confirmed by offline analysis. The level of systolic LVP at which the time constant $\tau$ was minimal in these patients was deemed to be the maximum tolerated systolic LVP in this subgroup.

In seven patients with $\mathrm{EF} \geq 0.30,500 \mathrm{~mL}$ of extracorporeal circulation priming solution were administered. Recordings of baseline and isovolumetric heartbeats were repeated after stabilization.

\section{Statistical analysis}

Quantitative results are presented as mean \pm SD. Systolic LVP is given in absolute values $(\mathrm{mmHg}$ ) or as a percentage of the isovolumetric pressure of each patient. Effects of LVP elevations were analysed with repeated measurements oneway ANOVA and Holm-Sidak post hoc test. Volume loading was compared to baseline with paired $t$ test. Linear regression was performed on normally distributed data by least squares regression and the Pearson correlation coefficient was obtained. Statistical significance was set at $P<0.05$.

\section{Results}

The mean patient age was $63 \pm 8$ years, 19 were men. Other preoperative data including drug therapy and comorbidities are summarized in Tables 1 and 2. These consecutive patients constitute a representative sample of CABG patients at our institution.

Individual haemodynamic data are presented in Supplemental Table 1. Patients were pooled in three groups according to their preoperative $\mathrm{EF}$ as assessed by $2 \mathrm{D}$ -
Table 1 Sample characteristics

\begin{tabular}{ll}
\hline Patient characteristics & $n=20$ \\
\hline Age & $63.2 \pm 8.0$ \\
Female gender & $1(5 \%)$ \\
BMI $\left(\mathrm{kg} / \mathrm{m}^{2}\right)$ & $27.0 \pm 2.6$ \\
Previous MI & $8(40 \%)$ \\
Diabetes mellitus & $2(10 \%)$ \\
Arterial hypertension & $12(60 \%)$ \\
COPD & $1(5 \%)$ \\
Haemoglobin $(\mathrm{g} / \mathrm{dL})$ & $13.9 \pm 1.58$ \\
Creatinine (mg/dL) & $1.00 \pm 0.29$ \\
Chronic medication & \\
$\beta$-Blockers & $15(75 \%)$ \\
ACEi/ARB & $10(50 \%)$ \\
Diuretics & $7(35 \%)$ \\
Nitrates & $16(84 \%)$ \\
CCB & $10(20 \%)$ \\
\hline
\end{tabular}

$B M I$ body mass index, $C O P D$ chronic obstructive pulmonary disease, $A C E i$ angiotensin converting enzyme inhibitors, $A R B$ angiotensin receptor blockers, $C C B$ calcium channel blockers

Table 2 Effects of volume loading in baseline and isovolumetric beats

Normal filling Volume loading

Baseline heartbeat

LVEDP ( $\mathrm{mmHg})$

$11.4 \pm 1.1 \quad 17.0 \pm 3.7 *$

$\mathrm{d} P / \mathrm{d} t_{\max }(\mathrm{mmHg} / \mathrm{s})$

$1363 \pm 241 \quad 1453 \pm 384$

$\mathrm{LVP}_{\max }(\mathrm{mmHg})$

$100 \pm 8$

$115 \pm 26^{*}$

$\tau(\mathrm{ms})$

$35.1 \pm 6.1 \quad 28.0 \pm 9.3^{*}$

Isovolumetric heartbeat

$\mathrm{LVP}_{\text {ISO }}(\mathrm{mmHg})$

$\tau_{\text {ISO }}$ (ms)

$207 \pm 77$

$220 \pm 53^{*}$

Time to $\mathrm{d} P / \mathrm{d} t_{\min }$ (ms)

$46.7 \pm 6.1$

$40.1 \pm 11.1^{*}$

Upward shift in LVEDP (mmHg)

$405 \pm 66$

$461 \pm 108^{*}$

$n=7$

LVEDP left ventricular end-diastolic pressure, $\mathrm{d} P / \mathrm{d} t_{\max }$ peak rate of left ventricular pressure rise, $L V P_{\max }$ maximum developed left ventricular pressure, $\tau$ time constant of isovolumetric relaxation, $L V P_{I S O}$ peak isovolumetric left ventricular pressure, $\tau_{I S O} \tau$ in the isovolumetric beat, $\mathrm{d} P / \mathrm{d} t_{\min }$ peak rate of left ventricular pressure fall $* P<0.05$ vs. normal filling by paired $t$ test

echocardiography (normal $\geq 0.50$; moderately decreased $0.30-0.49$; severely decreased $<30$ ). Twelve patients had a normal and eight patients a decreased EF. None of the patients with preserved ejection fraction presented heart failure signs or symptoms.

All patients underwent graded aortic constrictions resulting in beat-to-beat elevations of systolic LVP, ranging from a small elevation of a few $\mathrm{mmHg}$ to full isovolumetric beats. A representative LVP tracing illustrating the 
increase in LVEDP after complete aortic occlusion is presented in Fig. 1. Corresponding tracings from other patients are presented in Supplemental Fig. 1. Although there was no change in cycle length between the baseline and isovolumetric beats $(808 \pm 31$ vs. $805 \pm 21 \mathrm{~ms}$, respectively) the time to onset of pressure fall, as assessed by the time to $\mathrm{d} P / \mathrm{d} t_{\min }$, was delayed $(368 \pm 8$ vs. $435 \pm 14 \mathrm{~ms}, P<0.001$ ), denoting shorter time available to relax. Tracings, representative of each of the three EF patient categories, are presented in Fig. 2. In each panel, three pressure-time curves are superimposed: a control, an isovolumetric beat and one intermediate LVP elevation.

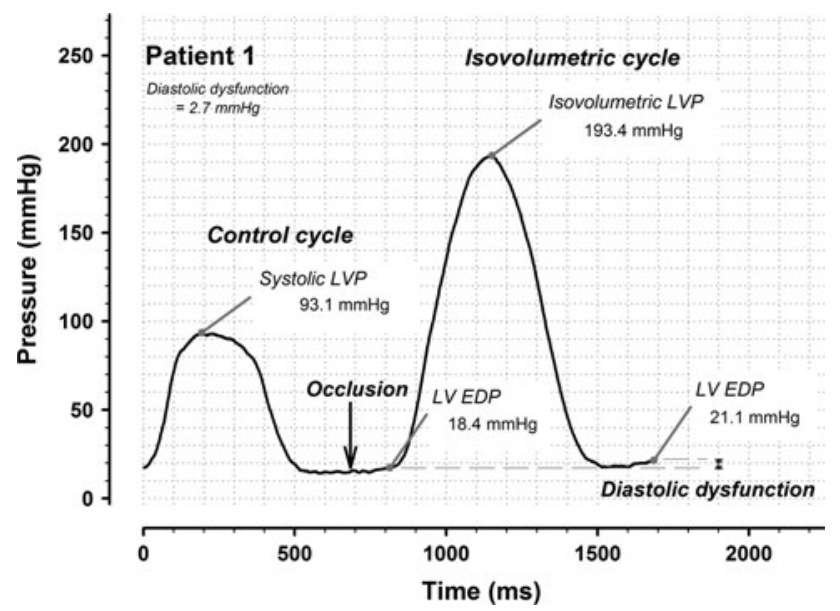

Fig. 1 A representative left ventricular pressure $(L V P)$ tracing of patient 1 illustrating the increase in left ventricular end-diastolic pressure ( $L V E D P)$ after complete aortic occlusion. LVP tracing of a control and isovolumetric cycle obtained in patient 1 after complete aortic occlusion during diastole. The upward shift in LV EDP from control to isovolumetric heartbeat, is represented
Peak isovolumetric LVP was higher in the left panel (normal EF) and lower in the right panel $(\mathrm{EF}<0.30)$. In the left panel, LVP elevations did not affect subsequent filling pressures, while the right panel, illustrates a patient with poor EF, who already presented elevated filling pressure at rest and showed further increases with both the intermediate and isovolumetric LVP elevations. As to the middle panel (EF 0.30-0.49), filling pressures were increased in the isovolumetric beat but not in the intermediate-afterloaded heartbeat. When we consider the patients with normal EF $(n=12)$, they operate at baseline (under anaesthesia) at a systolic pressure corresponding to $47.8 \pm 5.0 \%$ of peak isovolumetric pressure (Supplemental Table 2).

Increasing filling pressures, induced by beat-to-beat interventions, occurred without concomitant increases of diastolic volume in the recordings obtained with pressurevolume catheters, representing a true upward shift of the diastolic pressure-volume relation, hence afterload dependent diastolic dysfunction. Representative pressurevolume tracings are presented for a patient with an EF of 0.30-0.49 in Supplemental Fig. 2.

The shift in LVEDP induced by the first isovolumetric heartbeat after complete aortic occlusion was then correlated with haemodynamics and with LV function. Significant negative correlations were found with systolic function evaluated by $\mathrm{d} P / \mathrm{d} t_{\max }(r=-0.68)$ or peak isovolumetric LVP ( $r=-0.96$; Fig. 3, left panel). There was no correlation with the baseline value of the time constant $\tau$ or baseline LVEDP, but a close inverse correlation with the $\%$ change in $\tau$ induced by isovolumetric heartbeats $(r=$ 0.95; Fig. 3, right panel) was observed. Accordingly, the changes were more prominent in reduced EF categories.
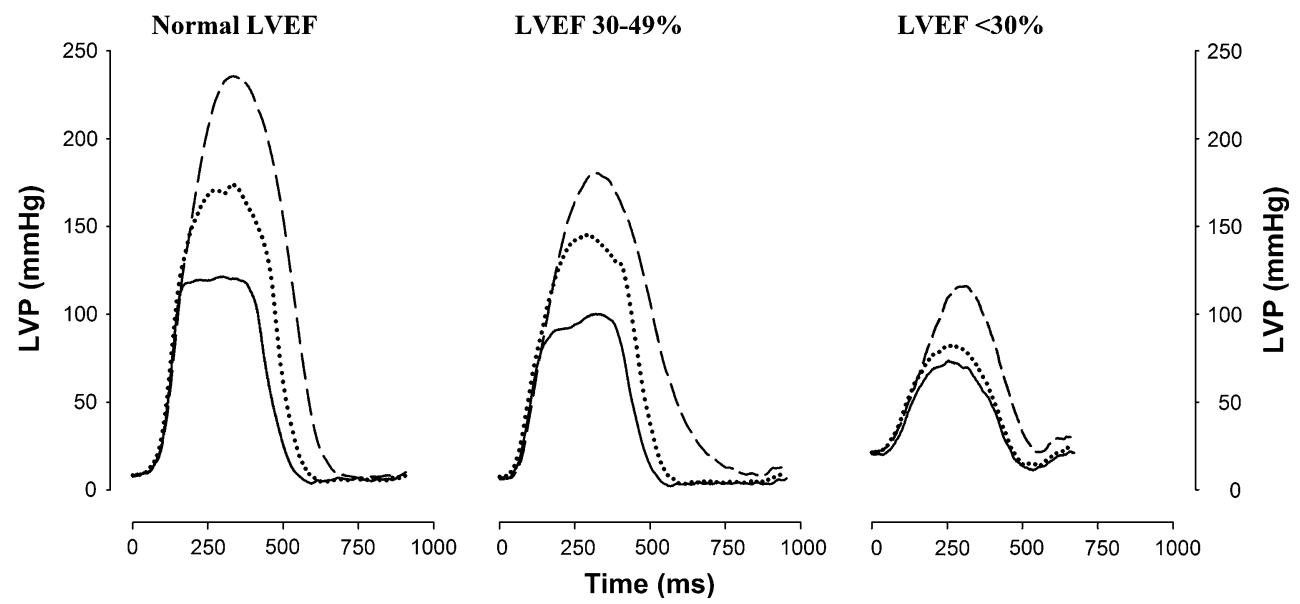

Fig. 2 The effects of left ventricular pressure $(L V P)$ elevation on diastolic pressures depend on systolic function. LVP tracings in baseline conditions (solid line), during a moderate LVP elevation (dotted line) and during an isovolumetric heartbeat (dashed line). Recordings of three representative patients are displayed: normal systolic function and normal ejection fraction (EF) on the left; moderately depressed systolic function and EF 30-49\% in the middle; severely depressed systolic function and $\mathrm{EF}<30 \%$ on the right. See text for details 


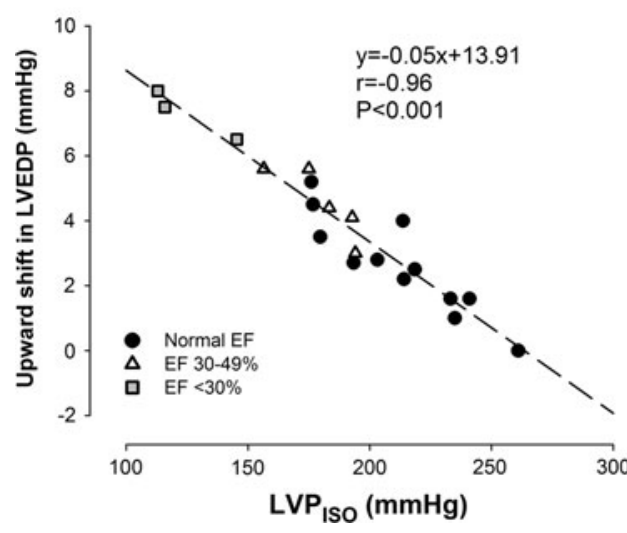

Fig. 3 The magnitude of the elevation of diastolic pressures closely correlates with systolic performance and with slowing of relaxation. The afterload-induced elevation of diastolic pressures, expressed as the upward shift of the left ventricular end-diastolic pressure $(L V E D P)$ is plotted as a function of the peak systolic pressure of an

For assessing the maximum systolic LVP that the heart could tolerate without slowing of relaxation and elevation of filling pressures, we analysed multiple-graded systolic pressures in each patient. Tolerated systolic LVP ranged from 60 to $100 \%$ of peak isovolumetric LVP (Supplement Table 3). In three patients with normal EF, afterload values of more than $230 \mathrm{mmHg}$ were tolerated. In three other patients with $\mathrm{EF}<0.30$, no pressure elevation was tolerated, even when baseline systolic LVP was lower than $90 \mathrm{mmHg}$. In these patients, the maximum tolerated systolic LVP was derived by caval occlusion (see "Materials and methods"). The remaining 14 patients tolerated intermediate levels of systolic LVP. Similarly to the magnitude of shift in diastolic pressure volume relation induced by isovolumetric heartbeats, tolerated systolic LVP was also strongly correlated with peak isovolumetric LVP $(r=0.99, P<0.001 ;$ Fig. 4$)$ and $\mathrm{d} P / \mathrm{d} t_{\max }(R=0.71$, $P<0.001$, not shown). Of note, the data of the three patients, in whom the maximum tolerated pressure was obtained with caval occlusion are well aligned with the other data.

In seven patients the experimental protocol was repeated after volume loading (Table 1). Volume loading increased diastolic and systolic LVP and accelerated myocardial relaxation (shortened time constant $\tau$ ). Peak isovolumetric LVP was higher after volume loading (Frank-Starling). Afterload-induced prolongation of the time constant $\tau$ was not exacerbated by volume loading, but the shift in LVEDP more than doubled. This is illustrated in Fig. 5, which shows in addition that, after volume loading, the onset of pressure fall was delayed (time to $\mathrm{d} P / \mathrm{d} t_{\min }$ increased, Table 1) and that, at matched HR, the duration of diastole decreased.

None of the patients died or suffered from neurological complications in the perioperative or early postoperative period (30 days). One patient received reintervention due

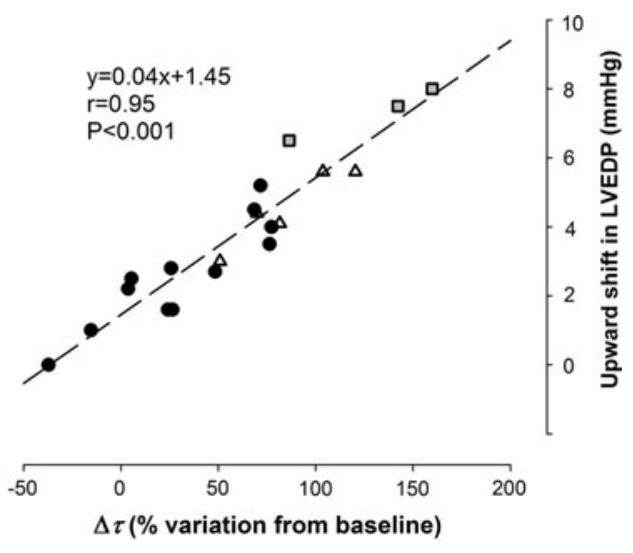

isovolumetric heartbeat $\left(L V P_{I S O}\right)$ (left panel) and as the percentage change of the logistic time constant $\tau$, induced by this isovolumetric beat (right panel). Symbols in gray scale correspond to different categories of ejection fraction $(E F)$, as indicated

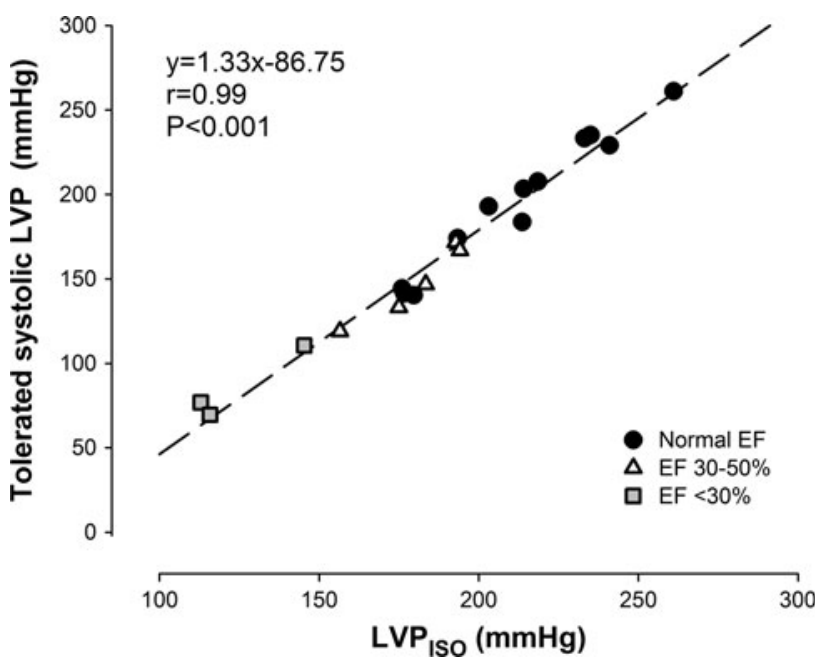

Fig. 4 Tolerated systolic left ventricular pressures (LVP) strongly correlate with peak isovolumetric LVP. Tolerated systolic LVP derived from the analysis of multiple-graded afterloaded heartbeats are plotted as a function of the peak systolic LVP of the isovolumetric beat $\left(L V P_{I S O}\right)$. Symbols in gray scale correspond to categories of ejection fraction $(E F)$, as indicated

to immediate postoperative bleeding, one patient needed continuous renal replacement therapy and two patients needed an intra-aortic balloon pump and prolonged cardiovascular support in the intensive care unit (ICU). The lengths of stay were $1.6 \pm 1.3$ and $8.0 \pm 3.9$ days in the ICU and in the hospital, respectively.

\section{Discussion}

The present investigation describes how human LV diastole responds to systolic pressure elevation, and defines for the first time how much systolic pressure is tolerated 


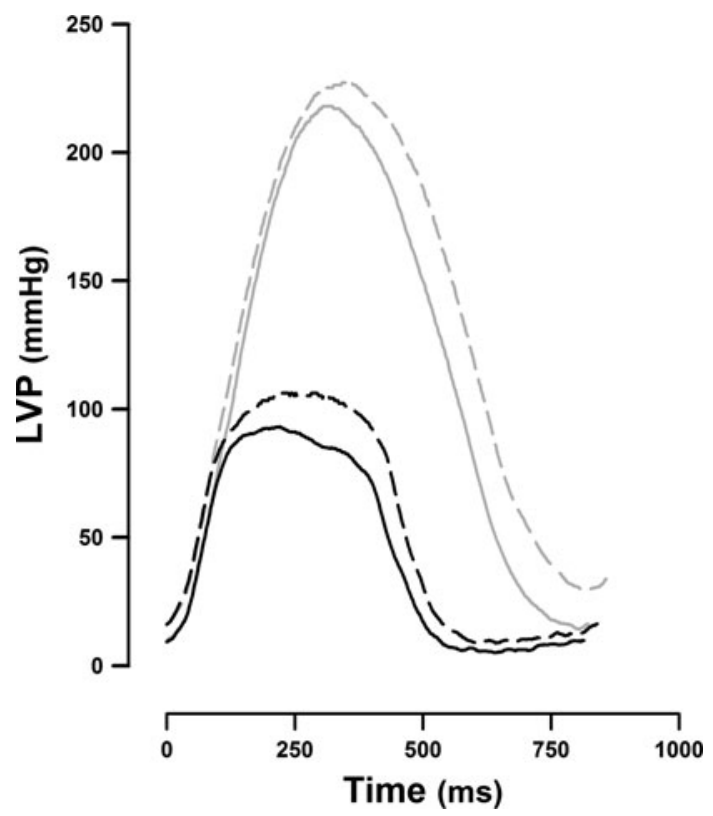

Fig. 5 Volume loading exacerbates afterload-induced diastolic dysfunction. Left ventricular pressure $(L V P)$ tracings of baseline (black lines) and isovolumetric heartbeats (grey lines).Data before (solid lines) and after (dashed lines) volume loading. After volume loading, pressure fall is delayed, but the rate of pressure fall is similar. Filling pressures rise much more in response to the isovolumetric condition after volume loading

without diastolic dysfunction. The study illustrates a close coupling between the diastolic response and systolic performance, evaluated by peak isovolumetric pressure.

In healthy animals, the physiological response of the myocardium to a moderate increase in afterload is a slight acceleration of relaxation [26] and no elevation of filling pressures [25]. This is valid up to a load level that corresponds to a high percentage of peak isovolumetric load $[8,26]$.

The analysis of isovolumetric heartbeats allows to refine earlier physiological observations and to apply them to patients with coronary heart disease. There is no precedent of a similar study conducted in humans, except maybe for the analysis of left ventricular systolic stiffness in seven patients by Ritter et al. [35]. From an ethical point of view it is important to note that we did not observe complications, carefully selected patients and performed intra-operative epivascular ultrasonography for excluding atherosclerotic aortic plaques. Given the increasing evidence of cerebrovascular complications with manipulations of the aorta, we however decided not to expand this series of patients. This is a "proof of concept", translational study, which allows us to draw some important conclusions even with 20 observations. This study describes how the better functioning human $\mathrm{LV}$ of CABG patients is able to develop a peak systolic LVP of more than $230 \mathrm{mmHg}$, with no slowing of relaxation and no increase in LVEDP, hence no diastolic dysfunction. Such a ventricle operates at rest at a systolic LVP that is $40-50 \%$ of peak isovolumetric LVP. This baseline load level corresponds to an optimal ventriculo-arterial hydraulic and energetic matching [6, 39]. It confers a surprisingly high afterload reserve to the healthy human LV, allowing it to face stress and exercise without compromising filling. This is the case even in the presence of severe coronary heart disease, during anaesthesia and surgery. The remaining patients responded to afterload with variable degrees of slowing of myocardial relaxation and diastolic dysfunction, manifest as an upward shift of the end-diastolic pressure-volume relation. This shift closely and inversely correlated with $d \mathrm{P} / d \mathrm{t}_{\max }$ and peak isovolumetric LVP: the better the systolic performance, the more limited the shift. In addition, this shift closely correlated with the changes in time constant $\tau$, suggesting that slowing of relaxation was responsible for the observed shift of the end-diastolic pressure-volume relation. This confirms in cardiac patients a close relation between systolic and diastolic function, previously described in various animal studies $[8,19,25]$. A limitation of the present study is that we performed volume measurements in only three patients. However, the effects of aortic clamping and the relation between delayed relaxation and increased filling pressures were also previously demonstrated in those animal models $[8,24,25]$.

By performing multiple-graded aortic constrictions, we determined the maximum level of systolic LVP that the heart could tolerate without elevating its filling pressures and slowing relaxation. The maximum tolerated LVP is highly predictive of the peak isovolumetric LVP and ranges from 60 to $100 \%$ of the isovolumetric LVP. The tolerated systolic LVP ranged from isovolumetric in some patients to baseline and even less in others. The observations on the maximum tolerated systolic pressures expand the knowledge on the effects of unphysiological isovolumetric pressures and provide a clinically useful translation of the concepts related to load-dependence of diastolic function. The findings are consonant with previous studies documenting in failing hearts the reversal of diastolic dysfunction in response to decreasing systolic pressures $[10,22]$. This was observed in heart failure patients with severely depressed systolic function (low EF's) [10], and in dogs with pacing-induced cardiomyopathy [22]. These findings imply that elevated filling pressures in patients with advanced HF with reduced EF include a load-dependent and potentially reversible component. A patient with a severely depressed EF and lower blood pressures works at a high percentage of isovolumetric load and mandatorily has load-dependent diastolic dysfunction, which can be limited with even small decreases of systolic pressure. This adds an additional pathophysiological mechanism for the beneficial effects of vasodilators and diuretics in this frequently occurring clinical condition. 
The effects of increased load on diastolic function were previously acknowledged by other groups. Vatner's group analysed the effects of increased systolic pressure and showed that in developing hypertensive heart disease in dogs, diastolic dysfunction was present. This dysfunction was not attributable to structural changes and could be mimicked in healthy animals by acutely increasing preload and afterload [18]. Similarly, aged dogs with renal hypertension and increased LV systolic and arterial stiffness presented impaired LV relaxation, but no increase in the coefficient of LV diastolic stiffness. Nevertheless, filling pressures increased with hypertensive episodes due to loaddependent impairment of relaxation [31].

Even if one should be aware of shifts in the end-diastolic pressure-volume relation, related to pericardial constraint and ventricular interdependence [1], these mechanisms were most likely not quantitatively important in the present open-chest and open-pericardium study conditions. Although these conditions might be well suited for the study of the pathophysiological effects of acute overload in the myocardium, they preclude extrapolation to long-term pressure elevations and to a clinical setting without anaesthesia and surgery.

By studying the effects of selective beat-to-beat afterload elevations on both relaxation rate and diastolic function, we were able to better control for confounding alterations of global haemodynamics due to decreased stroke volume, acute backward failure, increased LV filling pressures, neurohumoral responses and possibly afterload-induced myocardial ischemia [25]. Furthermore, as myocardial hypertrophy reduces tolerance to ischemia and coronary vasodilator reserve leading to diastolic dysfunction [16], we selected patients with a similar degree of stable 3-vessel coronary artery disease and excluded those with other than mild LV hypertrophy. Extent of myocardial ischemia and hypertrophy are therefore unlikely to have contributed significantly to diastolic dysfunction during transient aortic clamps with analysis of the first clamped heartbeat.

In seven patients with a normal or moderately depressed EF, afterload elevations were repeated after volume loading. Volume loading itself accelerated myocardial relaxation, which challenges earlier reports of slowed myocardial relaxation in volume-loaded anaesthetized open-chest dogs [34]. This observation is nevertheless consonant with what was shown in the better responding subgroup of coronary patients after leg elevation [9]. These apparently contradictory results might relate to anaesthesia and operative conditions of those earlier canine observations.

After volume loading, the upward shift of the end-diastolic pressure-volume relation was aggravated and this was not attributable to slower myocardial relaxation. This corroborates the recent demonstration in healthy dogs that changes in peak early-diastolic mitral annulus velocity $\left(e^{\prime}\right)$ are not dependent on myocardial relaxation, as assessed by time constant $\tau$, after acute preload manipulations [32]. Therefore, it must be due (at least in part) to prolongation of systole and abbreviation (at comparable HR) of the duration of diastole due to pressure and volume loading, as previously demonstrated in animal experiments [24]. Indeed, although acute afterload elevations also shorten diastole under normal filling conditions, this effect is exacerbated after volume loading. Other potential mechanisms still are largely speculative and require further investigation.

The negative inotropic effects of anaesthetics may have influenced the magnitude of effects, thus our results cannot be readily extended to unanaesthetized patients. The present study is applicable to patients with coronary heart disease with various EF's but not necessarily to other patients groups. Ischemia increases myocardial afterload sensitivity, even in hibernating and stunned myocardium [27, 36].

Though these were not included in our sample, the data could be relevant for patients with $\mathrm{HF}$ with preserved $\mathrm{EF}$ (HFpEF) in whom increased vascular load and ventricular systolic stiffness were shown to enhance the sensitivity of systolic pressures to volume changes [7]. Many patients with HFpEF have concomitant systolic dysfunction abnormalities, particularly in the long axis [4, 21, 28, 41], which may limit their tolerated systolic pressures. Beyond the profound structural changes underlying diastolic dysfunction and volume loading, limited tolerance to systolic pressures may provide additional mechanistic information on hypertensive pulmonary edema [15, 17].

In summary, the present manuscript extends and refines previous experimental work on load and diastolic function. The physiological response of the human heart to increased systolic pressures (and to volume loading) is the preservation of relaxation velocity and normal filling pressures. When cardiac function deteriorates, however, the LV becomes less tolerant to increased systolic pressures and reacts with slowed relaxation and increased filling pressures even at lower pressure levels. Volume loading further exacerbates such intolerance.

Acknowledgments This work was supported by the Portuguese Foundation for Science and Technology (grant number PIC/IC/82943/ 2007), through the Cardiovascular R\&D Unit (nr. 51/94) and by a grant from the European Commission (FP7-Health-2010; MEDIA261409).

\section{Conflict of interest None.}

\section{References}

1. Atherton JJ, Moore TD, Lele SS, Thomson HL, Galbraith AJ, Belenkie I, Tyberg JV, Frenneaux MP (1997) Diastolic ventricular interaction in chronic heart failure. Lancet 349:1720-1724. doi:10.1016/S0140-6736(96)05109-4 
2. Borbely A, Papp Z, Edes I, Paulus WJ (2009) Molecular determinants of heart failure with normal left ventricular ejection fraction. Pharmacol Rep. 61:139-145

3. Borlaug BA, Kass DA (2008) Ventricular-vascular interaction in heart failure. Heart Fail Clin 4:23-36. doi:10.1016/j.hfc.2007. 10.001

4. Borlaug BA, Lam CS, Roger VL, Rodeheffer RJ, Redfield MM (2009) Contractility and ventricular systolic stiffening in hypertensive heart disease insights into the pathogenesis of heart failure with preserved ejection fraction. J Am Coll Cardiol 54:410-418. doi:10.1016/j.jacc.2009.05.013

5. Borlaug BA, Melenovsky V, Redfield MM, Kessler K, Chang HJ, Abraham TP, Kass DA (2007) Impact of arterial load and loading sequence on left ventricular tissue velocities in humans. J Am Coll Cardiol 50:1570-1577. doi:10.1016/j.jacc.2007.07.032

6. Burkhoff D, Sagawa K (1986) Ventricular efficiency predicted by an analytical model. Am J Physiol 250:R1021-R1027

7. Chen CH, Nakayama M, Nevo E, Fetics BJ, Maughan WL, Kass DA (1998) Coupled systolic-ventricular and vascular stiffening with age: implications for pressure regulation and cardiac reserve in the elderly. J Am Coll Cardiol 32:1221-1227. doi:10.1016/ S0735-1097(98)00374-X

8. Correia-Pinto J, Henriques-Coelho T, Roncon-Albuquerque R Jr, Lourenco AP, Melo-Rocha G, Vasques-Novoa F, Gillebert TC, Leite-Moreira AF (2009) Time course and mechanisms of left ventricular systolic and diastolic dysfunction in monocrotalineinduced pulmonary hypertension. Basic Res Cardiol 104:535545. doi:10.1007/s00395-009-0017-3

9. De Hert SG, Gillebert TC, Ten Broecke PW, Mertens E, Rodrigus IE, Moulijn AC (1999) Contraction-relaxation coupling and impaired left ventricular performance in coronary surgery patients. Anesthesiology 90:748-757. doi:10.1097/00000542199903000-00017

10. Eichhorn EJ, Willard JE, Alvarez L, Kim AS, Glamann DB, Risser RC, Grayburn PA (1992) Are contraction and relaxation coupled in patients with and without congestive heart failure? Circulation 85:2132-2139. doi:10.1161/01.CIR.85.6.2132

11. Falcao-Pires I, Palladini G, Goncalves N, van der Velden J, Moreira-Goncalves D, Miranda-Silva D, Salinaro F, Paulus WJ, Niessen HW, Perlini S, Leite-Moreira AF (2011) Distinct mechanisms for diastolic dysfunction in diabetes mellitus and chronic pressure-overload. Basic Res Cardiol 106:801-814. doi: 10.1007/s00395-011-0184-x

12. Fukuta H, Little WC (2008) The cardiac cycle and the physiologic basis of left ventricular contraction, ejection, relaxation, and filling. Heart Fail Clin 4:1-11. doi:10.1016/j.hfc.2007.10.004

13. Gaasch WH, Delorey DE, Kueffer FJ, Zile MR (2009) Distribution of left ventricular ejection fraction in patients with ischemic and hypertensive heart disease and chronic heart failure. Am J Cardiol 104:1413-1415. doi:10.1016/j.amjcard.2009. 06.064

14. Gaasch WH, Little WC (2007) Assessment of left ventricular diastolic function and recognition of diastolic heart failure. Circulation 116:591-593. doi:10.1161/CIRCULATIONAHA.107. 716647

15. Gaasch WH, Zile MR (2004) Left ventricular diastolic dysfunction and diastolic heart failure. Annu Rev Med 55:373-394. doi: 10.1146/annurev.med.55.091902.104417

16. Galderisi M, Cicala S, Caso P, De Simone L, D'Errico A, Petrocelli A, de Divitiis O (2002) Coronary flow reserve and myocardial diastolic dysfunction in arterial hypertension. Am J Cardiol 90:860-864. doi:10.1016/S0002-9149(02)02708-X

17. Gandhi SK, Powers JC, Nomeir AM, Fowle K, Kitzman DW, Rankin KM, Little WC (2001) The pathogenesis of acute pulmonary edema associated with hypertension. N Engl J Med 344:17-22. doi:10.1056/NEJM200101043440103
18. Gelpi RJ, Pasipoularides A, Lader AS, Patrick TA, Chase N, Hittinger L, Shannon RP, Bishop SP, Vatner SF (1991) Changes in diastolic cardiac function in developing and stable perinephritic hypertension in conscious dogs. Circ Res 68:555-567. doi: 10.1161/01.RES.68.2.555

19. Gillebert TC, Leite-Moreira AF, De Hert SG (1997) Relaxationsystolic pressure relation. A load-independent assessment of left ventricular contractility. Circulation 95:745-752. doi:10.1161/01. CIR.95.3.745

20. Grossman W (2000) Defining diastolic dysfunction. Circulation. 101:2020-2021

21. Heusch G (2009) Diastolic heart failure: a misNOmer. Basic Res Cardiol 104:465-467. doi:10.1007/s00395-009-0025-3

22. Ishizaka S, Asanoi H, Wada O, Kameyama T, Inoue H (1995) Loading sequence plays an important role in enhanced load sensitivity of left ventricular relaxation in conscious dogs with tachycardia-induced cardiomyopathy. Circulation 92:3560-3567. doi:10.1161/01.CIR.92.12.3560

23. Ladeiras-Lopes R, Ferreira-Martins J, Leite-Moreira AF (2009) Acute neurohumoral modulation of diastolic function. Peptides 30:419-425. doi:10.1016/j.peptides.2008.10.018

24. Leite-Moreira AF, Correia-Pinto J (2001) Load as an acute determinant of end-diastolic pressure-volume relation. Am J Physiol Heart Circ Physiol 280:H51-H59

25. Leite-Moreira AF, Correia-Pinto J, Gillebert TC (1999) Afterload induced changes in myocardial relaxation: a mechanism for diastolic dysfunction. Cardiovasc Res 43:344-353. doi:10.1016/ S0008-6363(99)00099-1

26. Leite-Moreira AF, Gillebert TC (1994) Nonuniform course of left ventricular pressure fall and its regulation by load and contractile state. Circulation 90:2481-2491. doi:10.1161/01.CIR.90.5.2481

27. Leite-Moreira AF, Gillebert TC (1996) Myocardial relaxation in regionally stunned left ventricle. Am J Physiol 270:H509-H517

28. MacIver DH (2010) Is remodeling the dominant compensatory mechanism in both chronic heart failure with preserved and reduced left ventricular ejection fraction? Basic Res Cardiol 105:227-234. doi:10.1007/s00395-009-0063-x

29. Mann DL, Bristow MR (2005) Mechanisms and models in heart failure: the biomechanical model and beyond. Circulation 111:2837-2849. doi:10.1161/CIRCULATIONAHA.104.500546

30. Matsubara H, Takaki M, Yasuhara S, Araki J, Suga H (1995) Logistic time constant of isovolumic relaxation pressure-time curve in the canine left ventricle. Better alternative to exponential time constant. Circulation 92:2318-2326. doi:10.1161/01.CIR. 92.8.2318

31. Munagala VK, Hart CY, Burnett JC Jr, Meyer DM, Redfield MM (2005) Ventricular structure and function in aged dogs with renal hypertension: a model of experimental diastolic heart failure. Circulation 111:1128-1135. doi:10.1161/01.CIR.0000157183. 21404.63

32. Opdahl A, Remme EW, Helle-Valle T, Lyseggen E, Vartdal T, Pettersen E, Edvardsen T, Smiseth OA (2009) Determinants of left ventricular early-diastolic lengthening velocity: independent contributions from left ventricular relaxation, restoring forces, and lengthening load. Circulation 119:2578-2586. doi: 10.1161/CIRCULATIONAHA.108.791681

33. Paulus WJ, Grossman W, Serizawa T, Bourdillon PD, Pasipoularides A, Mirsky I (1985) Different effects of two types of ischemia on myocardial systolic and diastolic function. Am J Physiol 248:H719-H728

34. Raff GL, Glantz SA (1981) Volume loading slows left ventricular isovolumic relaxation rate. Evidence of load-dependent relaxation in the intact dog heart. Circ Res 48:813-824. doi: 10.1161/01.RES.48.6.813

35. Ritter M, Hess OM, Murakami T, Jenni R, Egloff L, Nonogi H, Schneider J, Krayenbuehl HP (1988) Left ventricular systolic 
series elastic properties in aortic stenosis before and after valve replacement. Cardiovasc Res 22:759-767. doi:10.1093/cvr/ 22.11.759

36. Schulz R, Rose J, Post H, Skyschally A, Heusch G (2000) Less afterload sensitivity in short-term hibernating than in acutely ischemic and stunned myocardium. Am J Physiol Heart Circ Physiol 279:H1106-H1110

37. Senzaki H, Kass DA (2009) Analysis of isovolumic relaxation in failing hearts by monoexponential time constants overestimates lusitropic change and load dependence: mechanisms and advantages of alternative logistic fit. Circ Heart Fail 3:268-276. doi: 10.1161/CIRCHEARTFAILURE.109.865592

38. Sossalla S, Maurer U, Schotola H, Hartmann N, Didie M, Zimmermann WH, Jacobshagen C, Wagner S, Maier LS (2011) Diastolic dysfunction and arrhythmias caused by overexpression of CaMKIIdelta(C) can be reversed by inhibition of late $\mathrm{Na}(+)$ current. Basic Res Cardiol 106:263-272. doi:10.1007/s00395010-0136-x

39. Suga H (1990) Ventricular energetics. Physiol Rev 70:247-277

40. Westermann D, Riad A, Richter U, Jager S, Savvatis K, Schuchardt M, Bergmann N, Tolle M, Nagorsen D, Gotthardt M, Schultheiss HP, Tschope C (2009) Enhancement of the endothelial NO synthase attenuates experimental diastolic heart failure. Basic Res Cardiol 104:499-509. doi:10.1007/s00395009-0014-6

41. Yip G, Wang M, Zhang Y, Fung JW, Ho PY, Sanderson JE (2002) Left ventricular long axis function in diastolic heart failure is reduced in both diastole and systole: time for a redefinition? Heart 87:121-125. doi:10.1136/heart.87.2.121 